\title{
Head and Neck Oncologic Surgery in the COVID-19 Pandemic: Our Experience in a Deep South Tertiary Care Center
}

\author{
Erin Buczek, $\mathrm{MD}^{1}$ \\ ${ }^{1}$ University of Alabama at Birmingham
}

April 29, 2020

\begin{abstract}
Introduction

The ongoing worldwide pandemic due to COVID-19 has forced drastic changes on the daily lives of the global population. This is most notable within the healthcare sector. The current paper outlines the response of the head and neck oncologic surgery (HNS) division within our academic otolaryngology department in the state of Alabama.

Methods

Data with regard to case numbers and types were obtained during the pandemic and compared with time matched data. Our overall approach to managing previously scheduled and new cases, personal protective equipment (PPE) utilization, outpatient clinic, and resident involvement is summarized.

Discussion

Our HNS division saw a 55\% reduction in surgical volume during the peak of the COVID-19 pandemic. We feel that an early and cohesive strategy to triaging surgical cases, PPE usage, and minimizing exposure of personnel is essential to providing care for HNS patients during this pandemic.
\end{abstract}

Morrison, Daniel R MD ${ }^{1 *}$, Gentile, Christopher $\mathrm{MD}^{1^{*}}$, McCammon, Susan $\mathrm{MD}^{1}$, Buczek, Erin $\mathrm{MD}^{1}$

Department of Otolaryngology, University of Alabama at Birmingham

*co-first authors

Corresponding Author:

Erin Buczek

Mailing Address:

Department of Otolaryngology - Head and Neck Surgery

$17202^{\text {nd }}$ Avenue $\mathrm{S}$

Birmingham, AL 35294-3412

Email: epartington@uabmc.edu

\section{Abstract}

Introduction

The ongoing worldwide pandemic due to COVID-19 has forced drastic changes on the daily lives of the global population. This is most notable within the healthcare sector. The current paper outlines the response of 
the head and neck oncologic surgery (HNS) division within our academic otolaryngology department in the state of Alabama.

\section{Methods}

Data with regard to case numbers and types were obtained during the pandemic and compared with time matched data. Our overall approach to managing previously scheduled and new cases, personal protective equipment (PPE) utilization, outpatient clinic, and resident involvement is summarized.

\section{Discussion}

Our HNS division saw a 55\% reduction in surgical volume during the peak of the COVID-19 pandemic. We feel that an early and cohesive strategy to triaging surgical cases, PPE usage, and minimizing exposure of personnel is essential to providing care for HNS patients during this pandemic.

\section{Introduction}

Severe acute respiratory syndrome coronavirus 2 (SARS-CoV-2), known colloquially as COVID-19, is a novel zoonotic infection that has caused a worldwide pandemic ${ }^{1}$. In many parts of the world the medical system has been severely stressed by the burden of this novel illness as the demand for medical care exceeds capacity. The illness is highly contagious and is spread through respiratory droplets. The highly contagious nature and relatively high mortality compared to other viral respiratory illnesses has made COVID-19 a "perfect storm" for a pandemic ${ }^{2}$. Otolaryngologists are at high risk for transmission of COVID-19 due to the high risk of contact with aerosolized nasal, pharyngeal, or tracheal secretions during many of our routine procedures ${ }^{3,4}$. The first reported physician death in Wuhan was an otolaryngologist ${ }^{5}$.

As of April 26, 2020 there were 6,213 confirmed COVID-19 cases in the state of Alabama (71,334 overall tested) with 213 reported deaths ${ }^{6}$. Of all patients tested, $8.7 \%$ were positive. For comparison, the state of New York, the hardest hit state in the United States thus far, had 282,143 total cases and 16,599 deaths $(777,568 \text { overall tested })^{7}$. $36.3 \%$ of tests given in NY state have been positive. Our institution is a large, tertiary care center situated in Birmingham, AL and receives patients from our entire state as well as portions of neighboring states (TN, MS, GA, FL). This is in contrast with NY, which has a much larger healthcare system, with multiple large hospital systems in the city of New York alone. Our relatively low overall case numbers compared with other regions are due to a multitude of reasons, including our relatively low population density, low amount of travel in and out of the state, and testing limitations. At our institution (University of Alabama-Birmingham), the number of COVID-19+ inpatients has not exceeded 62. We have had no documented positive COVID-19 tests among faculty, residents, advanced practice providers (APPs), or ancillary staff in our otolaryngology department. A focused timeline of events is shown in Figure 1.

There have been several recent publications focused on the response to COVID-19 within the otolaryngology community. Currently, the American Academy of Otolaryngology-Head and Neck Surgery (AAO-HNS) has recommended delaying all non-emergent operations and postponing all non-essential face-to-face clinic encounters ${ }^{8}$. Consistent with this is the recommendation that all tier 1 and 2 procedures as defined by the Centers for Medicaid and Medicare Services (CMS) should be deferred during the pandemic ${ }^{9,10}$. Early data from the pandemic also showed that cancer patients have significantly worse outcomes when infected with COVID-19 ${ }^{11}$. These data are to be interpreted with caution given possible regional differences in practice patterns with regard to resource utilization. With this context in mind, the current paper will retrospectively discuss the approach of the head and neck surgery (HNS) division of our tertiary care center in Birmingham, $\mathrm{AL}$ with regards to surgical care, outpatient care, and academics.

\section{Methods}

Current COVID-19 case numbers were reviewed nationally, statewide, and institutionally. Surgical volume was analyzed within the affected timeframe and compared with the year prior. As noted in Figure 1, hospital administration made the decision on March 17 to move to a limited OR model. Therefore, we selected March 18 as the starting point for our analysis and have analyzed up to April 21, 2020. Our approach to managing 
clinic outpatients is also reviewed as well as a brief overview of changes to resident involvement and inpatient management.

\section{Approach to Surgery}

Throughout February and early March, our department and hospital administration were closely monitoring the COVID-19 outbreak, however there were no changes to clinical operations. On March 16, one HNS case was cancelled due to blood shortages. UAB administration decided on March 17 to move to a limited operating room (OR) model, where only 6 ORs were to be opened daily, with 2 additional rooms open as needed for emergencies. Our freestanding outpatient surgical center was reduced to 3 working ORs with 1 available as needed for emergencies. At this point all elective cases were postponed indefinitely. For reference, our institution has 44 total ORs in our main hospital and 16 ORs at our outpatient center.

The HNS division began meeting virtually at this point to collectively triage cases. Remote conferences with this goal were held twice weekly during the limited OR model period. Cases were organized first into elective versus non-elective cases. Patients requiring non-elective surgery were defined as having a surgical disease process that will irreversibly worsen in a way that affects survival or extent of surgical intervention if surgical intervention were delayed. Elective cases were postponed indefinitely to be reassessed by each individual surgeon when conditions allow. Among the non-elective cases, 3 categories were described and are as follows: cases which should be done within 2 weeks, cases that should be done within $2-4$ weeks, and cases that are non-elective but can wait $>4$ weeks. These definitions were based on the likelihood of significant morbidity or mortality occurring within the defined time periods. For example, patients with significant airway concerns such as advanced laryngeal cancers and airway stenosis were placed in the 2 week category, while most thyroid malignancies were placed in the $>4$ week category (Figure 3 ).

Perioperative leadership including chairs of the surgical departments met to outline an appropriate triage strategy for operative cases. Per these institutional guidelines, each case scheduled required department chairman approval and was subject to review by the Chief of Surgical Services. This model remains in place. Our division now has a secure online calendar showing all HNS cases which is accessible by all faculty. This was used to streamline the chairman approval process. Figure 2 represents our case numbers during the time period under investigation. Of the utmost importance in triaging and completing these cases was a collective willingness in our division to cover cases for other surgeons in the case of mandated quarantines. Particularly early in UAB's response to the pandemic, pre-op testing was not available. During this time, we recognized there were approximately 5-10 patients that we considered urgent but also very high risk (endoscopic airway for stenosis, laryngeal carcinomas with airway compromise, etc.). We decided collectively that we would not perform these urgent but high risk cases without pre-op COVID testing or if not made available we would utilize all necessary PPE (see below) to perform these cases. Particularly, we met with anesthesia and operating room leadership to discuss the specific logistics of doing these cases. This decisive action led to early pre-op testing for our high risk patient population and likely saved the use of needed PPE.

Availability of COVID-19 testing nationwide has been a major issue during this pandemic. Initially, we were not able to preoperatively test our surgical patients. During this time, we were avoiding any procedure that risked exposure to aerosolized procedures such as tracheotomy and oral cavity cases. On March 28, our first preoperative COVID-19 tests were performed for planned operations on outpatients. Initially, we had only the capacity for 10 COVID-19 tests per day. Therefore, anesthesia staff selected the cases with highest risk exposures for preoperative testing. These patients were tested within 48 hours of their planned surgery. By April 6 we had increased our testing capacity and all surgical patients were tested once preoperatively within 48 hours of their surgery. All testing was RT-PCR via nasopharyngeal swab. At the current time, our institution is not using serologic testing to direct management.

Personal protective equipment (PPE) is paramount in protecting the surgeon as well as the patients and limiting disease spread. As the crisis progressed, we began concerted efforts to conserve essential PPE, most notably N95 respirators, gowns, gloves, and face shields. Although the data are conflicting regarding efficacy, N95 respirators are recommended for healthcare providers dealing with patients with unknown COVID-19 
status during aerosol generating procedures ${ }^{12,13}$. Per recommendations from the Stanford group we used tiered PPE depending on the case type ${ }^{14}$. Extreme airborne precautions (PAPR in addition to surgical gowns/gloves) was used for COVID positive cases and were considered high risk of transmission (upper aerodigestive tract, sinonasal cases, mastoidectomy). Enhanced airborne precautions (N95 respirator, eye protection, surgical gown/gloves) were used in COVID negative cases with high risk of transmission, any emergent case with unknown COVID status, urgent cases with COVID status unknown, positive symptoms or high risk cases. Standard PPE was used in low risk of transmission COVID negative cases and COVID unknown, asymptomatic patients undergoing urgent but low risk of transmission procedures. These guidelines are comparable to precautions outlined by other institutions ${ }^{15-18}$. Additionally, we have been extending the use of N95 respirators in accordance with the CDC's recommendations and prior data ${ }^{19,20}$. Our institution has been reprocessing N95 respirators for repeated use using a combination of UV light radiation and vaporized hydrogen peroxide. ${ }^{21-23}$.

Notably, once the decision was made to operate we did not alter our surgical approach for cases. Some data have shown a reduction or complete cessation in free flap surgeries during the pandemic at other institutions 24. Free tissue transfer and tracheostomies were still performed when indicated. We continued holding weekly multidisciplinary tumor board (MDTB), albeit this was done remotely via videoconferencing.

\section{Approach to Outpatient Clinics}

The multidisciplinary HNS clinic includes 8 specialists and 5 APPs and typically sees 1100 to 1200 new cancer cases every year. Beginning March $15^{\text {th }}$, a widespread clinic freeze was instituted by the department. Clinicians were asked to review their schedules for the next several weeks in order to identify patients on a case-by-case basis that could not be postponed. Patients were contacted individually by phone to reschedule although clinics were still open from an institutional standpoint. Until March $27^{\text {th }}$, clinics were continued on a limited basis and patients were limited to head and neck patients with an urgent need; new confirmed cancer diagnoses, post-op care, or patients with worsening symptoms due to their malignancy.

Beginning March $30^{\text {th }}$, all HNS clinics were consolidated to initially one clinic, one day per week. Due to volume, a second clinic day was added beginning April $6^{\text {th }}$. With the removal of dedicated clinic and OR block time, clinicians were able to remain flexible when covering the clinic days to allow for back up in the event of clinician exposure. Our department was fortunate to not have any confirmed cases of COVID-19 amongst the health care team, however one attending was symptomatic and subsequentially self-quarantined although ultimately tested negative. This clinician was able to continue to participate in telemedicine from quarantine.

Additionally, our department instituted a proactive and aggressive plan to pursue telemedicine for suitable patients to maintain continuity of care. It was left to the discretion of providers to determine patient's that would require in-person visits. As mentioned earlier, patients with a new cancer diagnosis, with recent surgery requiring wound checks or drain removals, or patients symptomatic with malignancy related symptoms were prioritized for in person clinic visits. When clinically appropriate, imaging was substituted for a physical exam to limit physical contact. While telemedicine certainly has its limitations, it also has notable advantages that merit discussion. Immunocompromised and medically infirm patients are able to stay at home and not risk exposure. There is more time for discussions regarding diagnoses, goals of care, etc. as well as a possibility of more frequent follow up. We anticipate that our practice patterns in the future will be adjusted to include more telemedicine as a result of this pandemic.

There continue to be limitations to this model. Telemedicine visits require patient access to an internet network or a "smartphone", limiting its use in some more isolated patient populations. Limiting the use of flexible scope exams in clinic and relying more heavily on imaging will likely have unforeseen consequences with regards to cancer surveillance, although this has yet to be seen and more data necessary to draw conclusions.

\section{Logistics and Resident education}


Outside of the clinic and OR setting, our department made several changes in the residency structure that will be detailed briefly. Institutionally, residents were not allowed to take care of COVID-19 positive patients or patients under investigation (PUIs). From a surgical standpoint, only one resident was allowed in a case at a time to conserve PPE. Beginning on March 19, the residency program was platooned into two groups, which alternated weekly shifts. The team that was not actively deployed at the hospital was responsible for continuing didactics and to remain on reserve in the event residents needed to be quarantined or became ill. During this time, the head and neck service accounted for two fellows, a senior level resident, and three junior level residents. Operative cases were performed with the least amount of personnel in the room, with the most experienced members performing the cases. Junior level residents (PGY 1-3) were generally not involved in operative cases during this time period unless needed. Many cases were done only by attending surgeons or by fellows. When junior residents were involved it was for assistant purposes only in order to maximize efficiency.

In attempts to minimize exposures, inpatient rounds were still performed by the resident teams with fellow assistance. Only senior residents examined inpatients and wore appropriate PPE depending on COVID status. If a patient had unknown status, a gown, gloves, and N95 respirator were worn. COVID+ patients were only seen in person by attending physicians wearing appropriate PPE (N95, gown, gloves, face shield).

By order of the Alabama governor, inpatient guest visitation was extremely limited. This hindered family education of trach care, wound care, use of nasogastric or gastrostomy tube, among other routine postoperative care. Residents were tasked with telephone updates following rounds. We anecdotally suspect this led to slightly longer inpatient stays due to the decreased hands-on training of family members. HNS patients are particularly reliant on family assistance after discharge. We did have some patients cancel their surgeries initially due to these restrictions. We ultimately were able to have family members receive in-person teaching towards the end of the hospital stay. A "compassionate" exception was made for patient with dementia or other factors requiring close caregiver participation (this was allowed with two patients). Another unforeseen circumstance of the visitation restrictions was the occasional cancellation of urgent cases by patients. In addition, patients were not routinely screened post-operatively for COVID-19 unless they began to develop symptoms.

\section{Discussion}

At the current time (April 26, 2020), there are 943,865 cases of COVID-19 in the United States, 6,213 of which are in Alabama ${ }^{6}$. For comparison, surrounding southern states Louisiana, Georgia, and Mississippi have 26,512, 23,401, and 5718 cases respectively while New York has 282,143 cases $^{25}$. These numbers highlight the variability to which the pandemic has affected different states thus far, even within the same geographic region. New York State contains 411.2 people per square mile compared to 168 people per square mile of neighboring Georgia, 94.4 people per square mile in Alabama and 63.2 people per square mile in Mississippi ${ }^{26}$. While there are many factors involved, the overall low population density, early mitigation in Alabama's largest urban area (Birmingham), and limitations in testing have contributed to lower COVID-19 case numbers.

Due to the trajectory and distribution of new COVID-19 cases, our department has been able to implement strategies used in other states and countries ${ }^{15,16,18,27-30}$. The authors would direct you to Patel et al. for a recent description of HNS practice patterns nationwide during the pandemic ${ }^{24}$. Since the beginning of the pandemic, the maximum number of COVID-19 patients at our institution has not exceeded 62 and therefore, our institution has not yet experienced ventilator or PPE shortages suffered by other health care centers. We feel that thus far our division has been able to effectively triage HNS patients and complete their oncologic surgeries safely, while simultaneously reducing non-urgent surgeries. We reduced our surgical volume by $55 \%$ during the critical time period and this was predominantly by postponing elective, outpatient surgeries. Of note, we feel strongly that a team-based approach toward completing surgical cases is of the utmost importance in this situation. This requires inter-provider consistency with regard to management strategies, a high level of communication, and an understanding between the patient and provider that another surgeon may be involved or primarily responsible for their care. A foundation of trust between the patient and 
provider is elemental in the success of this model. We would advocate a high level of detail with regard to documentation and completion of the surgical consent with these principles in mind.

An early and constantly evolving response to the COVID-19 crisis remains critical to avoid an overwhelming surge on our medical system. At this time, ORs both at our institution and around the country are beginning to re-open to clear the logjam of semi-urgent and elective cases. We feel the keys to safely moving forward with increased head and neck surgical volume is with widespread, reliable pre-operative COVID testing, adequate PPE and a clear mechanism to triage cases according to urgency and safety via a team-based approach. While our experience may not be reproducible for smaller community hospitals or tertiary centers located in more urban settings, they do serve to highlight the response and experience of a high-volume head and neck cancer tertiary center situated in the deep South.

\section{Literature Cited}

1. Huang C, Wang Y, Li X, et al. Clinical features of patients infected with 2019 novel coronavirus in Wuhan, China. Lancet.2020;395(10223):497-506.

2. Medicine JHUo. COVID-19 Dashboard by the Center for Systems Science and Engineering (CSSE) at Johns Hopkins University. https://coronavirus.jhu.edu/map.html. Published 2020. Accessed 2020, April 26.

3. Meng L, Hua F, Bian Z. Coronavirus Disease 2019 (COVID-19): Emerging and Future Challenges for Dental and Oral Medicine. J Dent Res.2020;99(5):481-487.

4. Zou L, Ruan F, Huang M, et al. SARS-CoV-2 Viral Load in Upper Respiratory Specimens of Infected Patients. N Engl J Med.2020;382(12):1177-1179.

5. Chan JYK, Wong EWY, Lam W. Practical Aspects of Otolaryngologic Clinical Services During the 2019 Novel Coronavirus Epidemic: An Experience in Hong Kong. JAMA Otolaryngol Head Neck Surg. 2020.

6. Health ADoP. Alabama's COVID-19 Data and Surveillance Dashboard. https://alpublichealth.maps.arcgis.com/apps/opsd Published 2020. Accessed 2020, April 22.

7. Health NYSDo. NYSDOH COVID-19 Tracker. https://covid19tracker.health.ny.gov/views/NYS-COVID19Tracker/NYSDOHCOVID-19Tracker-Map?\%3Aembed=yes\&\%3Atoolbar=no\&\%3Atabs=n. Published 2020.

Accessed 2020, April 23.

8. Surgery AAoO-HaN. New Recommendations Regarding Urgent and Nonurgent Patient Care. https://www.entnet.org/cont recommendations-regarding-urgent-and-nonurgent-patient-care. Published 2020. Accessed.

9. Bann DV, Patel VA, Saadi R, et al. Impact of coronavirus (COVID-19) on otolaryngologic surgery: Brief commentary. Head Neck. 2020.

10. Services CfMaM. Non-Emergent, Elective Medical Services, and Treatment Recommendations. https://www.cms.gov/files non-emergent-elective-medical-recommendations.pdf. Published 2020. Updated 2020, April 7. Accessed 2020, April 23.

11. Liang W, Guan W, Chen R, et al. Cancer patients in SARS-CoV-2 infection: a nationwide analysis in China. Lancet Oncol.2020;21(3):335-337.

12. Smith JD, MacDougall CC, Johnstone J, Copes RA, Schwartz B, Garber GE. Effectiveness of N95 respirators versus surgical masks in protecting health care workers from acute respiratory infection: a systematic review and meta-analysis. CMAJ. 2016;188(8):567-574.

13. Long Y, Hu T, Liu L, et al. Effectiveness of N95 respirators versus surgical masks against influenza: A systematic review and meta-analysis.J Evid Based Med. 2020.

14. Vukkadala N, Qian ZJ, Holsinger FC, Patel ZM, Rosenthal E. COVID-19 and the Otolaryngologist: Preliminary Evidence-Based Review.Laryngoscope. 2020. 
15. Vukkadala N, Qian ZJ, Holsinger FC, Patel ZM, Rosenthal E. COVID-19 and the otolaryngologist preliminary evidence-based review. The Laryngoscope. 2020.

16. Day AT, Sher DJ, Lee RC, et al. Head and neck oncology during the COVID-19 pandemic: Reconsidering traditional treatment paradigms in light of new surgical and other multilevel risks. Oral Oncology.2020;105:104684.

17. Pichi B, Mazzola F, Bonsembiante A, et al. CORONA-steps for tracheotomy in COVID-19 patients: A staff-safe method for airway management. Oral oncology. 2020;105:104682-104682.

18. Zimmermann M, Nkenke E. Approaches to the management of patients in oral and maxillofacial surgery during COVID-19 pandemic. Journal of Cranio-Maxillofacial Surgery. 2020.

19. Bergman MS, Viscusi DJ, Zhuang Z, Palmiero AJ, Powell JB, Shaffer RE. Impact of multiple consecutive donnings on filtering facepiece respirator fit. Am J Infect Control. 2012;40(4):375-380.

20. Control CfD. Strategies for Optimizing the Supply of N95 Respirators. https://www.cdc.gov/coronavirus/2019ncov/hcp/respirators-strategy/index.html. Published 2020. Accessed April 23, 2020.

21. Mills D, Harnish DA, Lawrence C, Sandoval-Powers M, Heimbuch BK. Ultraviolet germicidal irradiation of influenza-contaminated N95 filtering facepiece respirators. Am J Infect Control.2018;46(7):e49-e55.

22. Mackenzie D. Reuse of N95 Masks. Engineering (Beijing).2020:10.1016/j.eng.2020.1004.1003.

23. Schwartz A, Stiegel M, Greeson N, et al. Decontamination and Reuse of N95 Respirators with Hydrogen Peroxide Vapor to Address Worldwide Personal Protective Equipment Shortages During the SARS-CoV-2 (COVID-19) Pandemic. Applied Biosafety. 2020:1535676020919932.

24. Patel RJ, Kejner A, McMullen C. Early Institutional Head and Neck Oncologic and Microvascular Surgery Practice Patterns Across the United States During the SARS-CoV-2 (COVID19) Pandemic. Head Neck. 2020.

25. Centers for Disease Control and Prevention. Cases of coronavirus disease (COVID-19) in the U.S. https://www.cdc.gov/coronavirus/2019-ncov/cases-updates/cases-in-us.html\#1. Published 2020. Updated April 23. Accessed April 26, 2020.

26. Bureau USC. 2010 Census: Population Density Data. https://www.census.gov/data/tables/2010/dec/densitydata-text.html. Published 2020. Updated May 4, 2018. Accessed April 26, 2020.

27. Givi B, Schiff BA, Chinn SB, et al. Safety Recommendations for Evaluation and Surgery of the Head and Neck During the COVID-19 Pandemic. JAMA otolaryngology-head E neck surgery. 2020.

28. Kettering CSotORECaMS. Cancer Surgery and COVID19. Ann Surg Oncol. 2020:1-4.

29. Ansarin M. Surgical management of head and neck tumours during the SARS-CoV (Covid-19) pandemic. Acta Otorhinolaryngol Ital. 2020.

30. Boccalatte LA, Larrañaga JJ, Perez Raffo GM, et al. Brief guideline for the prevention of COVID-19 infection in head and neck and otolaryngology surgeons. American Journal of Otolaryngology.2020:102484.

\section{Hosted file}

COVID-19_figures.docx available at https ://authorea.com/users/312328/articles/446530-head-andneck-oncologic-surgery-in-the-covid-19-pandemic-our-experience-in-a-deep-south-tertiary-carecenter 